\title{
La formación y capacitación política en la Argentina democrática
}

Political education and training in democratic Argentina

María Melina Guardamagna Universidad Nacional de Cuyo. meliguardamagna@gmail.com.
Fecha de recepción 13.11.13

Fecha de aceptación 21.2.14

\section{Resumen}

La preocupación por formar cuadros políticos para la gestión del Estado adquiere rango constitucional en 1994 cuando el Estado se compromete a contribuir a la capacitación de los dirigentes de los partidos políticos. Para la implementación de dicha política se crearon dos instituciones de formación y capacitación político estatales: la Escuela Nacional de Gobierno y el Instituto de Capacitación y de Dirigentes Políticos. El análisis del proceso de formulación e implementación de la política busca comprender el devenir de estas instituciones que, desde su creación, fueron cerrándose cada vez más a las necesidades coyunturales de cada gestión, generando la gubernamentalización de componentes estatales e impidiendo la estabilización de un modelo de formación y capacitación de cuadros políticos tal como lo prescribe la Constitución $\mathrm{Na}$ cional en su artículo 38.

Palabras clave: Estado - política - formación cuadros políticos - democracia.

\section{Abstract}

Concern on the development of politically motivated cadres devoted to State management reached constitutional rank in 1994, when the State undertakes the commitment to contribute to the training of the leaders of political parties. Two public institutions were thus enacted: the National School of Government and the Institute for 
the Training of Political Leaders. The analysis of the process of formulation and implementation of policy seeks to understand the evolution of these institutions which, since its inception, were progressively reoriented towards a short-term approach linked to the successive governmental periods, in an implicit reduction of the State to the Executive branch which contrasts with the constitutional provisions of Article 38.

Key word: State - policy - training - political cadres - democracy.

\section{Introducción $^{1}$}

La formación y capacitación de cuadros políticos para la gestión del Estado constituye un preocupación que atraviesa tanto a la teoría como a la práctica política y que, por lo tanto, se trata de una cuestión que es necesario abordar desde ambas ópticas. Sin embargo, en el caso argentino, este no ha sido un tema de preocupación del ámbito académico, siendo escasos los estudios dedicados al abordaje de la problemática. El presente trabajo pretende constituir un aporte al análisis de las políticas implementadas por el Estado argentino orientadas a la formación y capacitación de cuadros políticos en democracia, específicamente desde la incorporación del artículo 38a la Constitución Nacional en $1994 .^{2}$

La identificación, caracterización y análisis de las sucesivas políticas implementadas por el Estado argentino a través de la Escuela Nacional de Gobierno (ENG) y del Instituto Nacional de Capacitación Política (INCaP) del Ministerio del Interior, junto a la interpretación y comprensión del proceso de formulación e implementación de la política orientada a la formación y capacitación de cuadros políticos constituye, de esta manera, un aporte indispensable en la búsqueda de diversas y posibles soluciones a un problema aún pendiente para los argentinos: la formación y capacitación de nuestra clase dirigente.

El abordaje de la política que constituye el objeto de estudio de esta investigación, por las agencias estatales mencionadas, se realizó a través de un estudio longitudinal desde una estrategia de tipo cualitativa e identificando sucesivamente cada uno de los siguientes períodos de gobierno: la gestión de Carlos Menem desde 1994 a 1999; la de Fernando De la Rúa (1999-2001); la de Eduardo Duhalde (2002-2003); la de Néstor Kirchner (2003-2007) y la de Cristina Fernández de Kirchner (2007-2011). 
Las componentes de la política analizadas en cada uno de estos momentos se desprenden de una matriz teórico-analítica (Cuadro 1) construida a partir del enfoque propuesto por Oszlak y O’Donnell (1976). Como se resume en el Cuadro 1 del Apéndice, son las siguientes:

1. Problematización del asunto, que en este caso se refiere al momento histórico en el cual se explicita por primera vez la necesidad de una política estatal destinada a la formación y capacitación de los cuadros dirigentes del Estado; las ideas sobre las que se justificó dicha política y las instituciones a través de las cuales se implementó la misma;

2. Contexto, tanto de las ideas como el institucional, en el que dicho asunto comienza a integrar la agenda de cuestiones socialmente problematizadas y los diversos contextos en los que, a lo largo de cada período de estudio, se redefine la política;

3. Forma en que se definió la cuestión en el momento del surgimiento histórico de la misma y su redefinición en cada uno de los períodos democráticos seleccionados. Nos preguntamos aquí cómo, en cada uno de estos momentos, el Estado logró construir consenso social en torno a la necesidad de contar con cuadros políticos formados para la gestión y cómo definió el perfil de los mismos. Para ello utilizamos la clasificación propuesta por Carlos Matus (2008), quien distingue entre cabecillas, políticos y estadistas.

A los fines de nuestro análisis interesan los perfiles del político y del estadista, ya que la mayoría de sus capacidades pueden ser adquiridas a través de procesos de formación y capacitación. Tal como sostiene Matus, el poder de un actor político se compone de cinco fuentes: la personalidad, la motivación, el control de los recursos, la experticia con que administra los recursos que controla y el soporte técnico-cognitivo, es decir, el valor científico-técnico de las herramientas que maneja y controla (2008: 65). Las dos primeras fuentes se relacionan con atributos propios de la persona que difícilmente serán aprehendidos mediante instancias formales de capacitación. El patrimonio de recursos que el líder posee o controla, ya sea en forma directa como control de recursos o indirectamente como soporte y adhesiones de otros actores sociales, es el que le permite al líder "producir decisiones y acciones frente a los otros actores sociales que participan en el juego social” (íd 2008: 69).

La capacidad del político para la toma de decisiones tiene que ver con la experticia con que administra dichos recursos. "La experticia de gobierno tiene como base el capital experiencia ponderado por el capital intelectual, incluido el capital ideológico, con que dicha experiencia se acumula. Si la experiencia es cero, por muy alto que sea el capital intelectual, la experticia es cero. De la misma manera, si el capital intelectual es cercano a cero, por muy prolongada que se la experiencia, la experticia es cercana a cero" (Matus 2008: 75). De esta forma, el político acumula experticia cuando tiene una base teórica a partir de la cual interpreta la realidad. Finalmente, el soporte técnico-científico le permite al político diferenciar el valor relativo científico-técnico de cada uno de los re- 
cursos que posee. Es decir que "cada control de un recurso debe ponderarse por su valor relativo técnico-científico"; este conjunto de capacidades personales, aunque presentan cierta rigidez, admite "un proceso significativo de aprendizaje" (íd. 77).

4. Toma de posición del Estado, es decir la política formulada e implementada por los diversos gobiernos tendiente a resolver el problema de la formación y capacitación de los cuadros políticos y las ideas a partir de las cuales se ha fundamentado en cada momento; 5. Actores que se involucran en torno a la cuestión y la forma en que influye la toma de posición estatal respecto a la de estos otros actores sociales;

6. Recursos y apoyos que los actores movilizan para la resolución de la cuestión;

7. Cristalizaciones institucionales que se producen en el proceso de resolución de la cuestión, es decir, las unidades burocráticas a través de las cuales el Estado implementa la política en cada período y las consecuencias que dichas cristalizaciones pueden tener respecto de futuras tomas de posición por parte del Estado.

8. El modo dominante mediante el cual se intenta resolver la cuestión en cada momento;

9. Cambios sociales e internos al Estado que puedan ser atribuibles al proceso generado en torno a la política. Es decir, cuáles han sido las contribuciones del Estado, producto de la implementación de la política, al cambio social, si la política ha alentado la generación de valores tendientes al fortalecimiento de la democracia y, en tal caso, si dichos valores han modificado la relación entre gobernantes y gobernados.

Se trata de componentes que permitieron el análisis del proceso de formulación e implementación de la política estatal en tanto expresión dinámica del Estado. Abordaje que, al mismo tiempo, se entrecruza, con otras expresiones de lo estatal, es decir, el Estado como relación social y como conjunto de instituciones a través de las cuales se materializa.

Para el desarrollo de la investigación se utilizaron métodos y técnicas cualitativas como el análisis documental (leyes, resoluciones, decretos, diarios de sesiones, publicaciones, programas, artículos periodísticos, investigaciones académicas, etc.) y la realización de entrevistas en profundidad a actores claves participantes de los procesos de toma de decisiones en la política objeto de estudio. Para ello, se operacionalizó un cuestionario, semi-estructurado, con preguntas representativas de la matriz teórico-analítica. De esta forma, la conjunción de los métodos de análisis documental y de entrevistas en profundidad permitió triangular los dichos de los entrevistados con el análisis de la información a fin de comprender e interpretar más acabadamente el fenómeno en estudio, contribuyendo, en la medida de lo posible, a delinear un modelo capaz de trascender las gestiones de gobierno, teniendo en cuenta las condicionalidades que presenta el sistema político y social argentino. 


\section{La formación y capacitación de cuadros políticos en el Estado}

En la historia contemporánea argentina hay un momento del desarrollo político estatal en el que se explicita abiertamente la necesidad de una política de formación y capacitación para sus cuadros dirigentes, que hasta entonces no se había formulado. A partir de allí emerge la necesidad y se formaliza una política que más allá de integrar la agenda de cuestiones socialmente problematizadas, no logra estabilizarse y alcanzar altos niveles de institucionalización, quedando atrapada en la discusión de quién y dónde se tienen que concretar esos procesos de formación y capacitación: ¿Es en las universidades estatales y/o privadas? ¿Es en los partidos políticos? ¿Es en organizaciones de la sociedad civil? ¿Es en los sindicatos? ¿O es simplemente desde el propio Estado a través de agencias creadas para tal fin?

En la década de 1930 la búsqueda de salida de la crisis mundial pone al Estado en el centro de la escena. Junto a la instauración del Estado de Bienestar se empieza a visualizar que el Estado es el actor central de la política y a descubrir que no está pensado ni diseñado para actuar como tal. El nuevo rol intervencionista del Estado requiere una clase dirigente capaz de cumplir los objetivos de la política nacional. Los herederos de la Generación del 80, aglutinados en el Partido Conservador, extraños a los intereses de la mayoría y a las crecientes demandas provenientes de los sectores más sensibles de la sociedad, no habían tenido la necesidad de formar dirigentes políticos capaces de dar respuesta a estas demandas, ya que hasta entonces la elite gobernante había estado conformada por los mismos individuos que integraban las elites económicas del país. Sin embargo, en la década de 1940 los grupos que hasta entonces habían gobernado el país, pierden legitimidad para conducir el proyecto nacional.

En este contexto, el gobierno de Juan Domingo Perón significó un quiebre en el razonamiento de cómo debía ser el Estado y cómo debían formarse y capacitarse los cuadros políticos que iban a conducir ese Estado, siendo tan imperiosa esta necesidad que se plantea en el texto constitucional de 1949. De esta forma, lo que hizo el peronismo a través del artículo 37 de la Constitución Nacional (CN) fue cristalizar una cuestión que ya había empezado a problematizarse en las dos últimas décadas: la necesidad de dar formación política a quienes se encargarán de la conducción del Estado. A partir de ese momento dicha preocupación queda instalada en el Estado, pero siempre inconclusa, nunca suficientemente resuelta, nunca adecuadamente diagnosticada y sin certezas de lo que se quería formar y capacitar. Se transforma en una cuestión que aparece de manera discontinuada como un elemento más de las propias crisis que atraviesa el desarrollo del Estado argentino.

El artículo 37 de la Constitución de $1949^{3}$ enunció una política de formación y capacitación de dirigentes políticos que se fundamentaba teóricamente en los principios de la 
doctrina peronista y en la idea de impulsar un programa educativo orientado a la inclusión y movilidad social a través del cual se lograría la revolución política y cultural. Se buscaba formar una élite política plural, razón por la que se involucró a las universidades.

Los primeros intentos de implementación de la política se realizaron en 1950, con cursos de formación política a dictarse en las universidades estatales. Sin embargo, el clima antiperonista que reinaba en la mayoría de ellas dificultó la efectiva y generalizada ejecución de la misma. En la Universidad de Buenos Aires los alumnos, en su mayoría opositores al gobierno, impidieron el dictado de los cursos, tal como Tulio Halperín Donghi (2008) lo relata en sus memorias.

En la Universidad Nacional de Cuyo (UNCuyo), comenzaron a dictarse cursos generales y obligatorios de formación política para el conocimiento de la realidad argentina, que anticiparon la creación de una carrera de estudios universitarios y de una unidad académica específica para el desarrollo de la ciencia política regional. Los cursos no sólo se orientaron a la "formación de especialistas para la alta burocracia del Estado", sino también a "dirigentes sindicales y de partidos políticos, formadores de opinión pública y funcionarios capacitados para la dirección de las empresas públicas estatales" (Sampay 1951: 18). A través del artículo 37 CN se pensó una instancia de capacitación política destinada a los estudiantes de todas las carreras que se dictaban en la universidad. Sin embargo esto nunca se cumplió, ya que el Estado terminó impulsando la creación de una unidad de formación específica para el estudio de la política y lo social, como fue la Escuela de Estudios Políticos y Sociales que en el año 1967 adquiriría el rango de Facultad de Ciencias Políticas y Sociales en la UNCuyo. De esta forma, la lógica de la organización universitaria que supone la disposición de los saberes en unidades académicas específicas, terminó reinterpretando la política del gobierno de Perón. Con el tiempo, quienes no optaron por dicha unidad académica, no tuvieron formación política. Algo que años más tarde, también sucedería a partir de lo dispuesto por el artículo 38 de la Constitución Nacional de 1994 y la creación de instituciones como la Escuela Nacional de Gobierno (ENG) y el Instituto de Capacitación y Formación de Dirigentes Políticos que, durante la presidencia de Eduardo Duhalde pasaría a llamarse Instituto Nacional de Capacitación de Dirigentes Políticos y Sociales; a partir de 2004 se denominó Instituto Nacional de Capacitación Política (INCaP), que desde entonces centralizan la formación y capacitación de la clase dirigente en la órbita del Estado.

A mediados de la década de 1950 varias universidades privadas confesionales fueron fundadas y muchas de ellas crearon Departamentos de Ciencia Política dentro de las Facultades de Ciencias Sociales, con el fin de formar dirigentes católicos. Por medio del artículo 28 del Decreto-Ley 6.403 del Poder Ejecutivo Nacional (diciembre de 1955), el gobierno de la "Revolución Libertadora" autorizó la creación de universidades privadas; artículo que recién sería reglamentado durante la presidencia de Arturo Frondizi, 
en medio de profundos enfrentamientos y de las presiones de la Iglesia. "Entre los sectores que apoyaron el golpe de estado de 1955, también estaban aquellos que, como la Iglesia, solicitarían se contemplaran sus intereses en un área decisiva para la formación de dirigentes y para la expansión de una influencia social que, con razón, se consideraba no representada en el cuerpo de universidades públicas. El artículo 28, que habilita las universidades privadas en condiciones de competir con las públicas, es una respuesta a estas reivindicaciones" (Sarlo 2007: 88). Bajo estas premisas, a partir de 1965 comienza a funcionar en el ámbito de la Universidad Católica Argentina (UCA) la Escuela de Ciencias Políticas como escuela de postgrado, ámbito en el cual se cursaba un Doctorado en Ciencias Políticas. Siete años después se abrió la Licenciatura en Ciencias Políticas que apuntaba al mismo objetivo que se había trazado el curso inicial de doctorado, esto es, la formación de vocaciones políticas, de diplomáticos y de funcionarios públicos.

El período iniciado a mediados de la década de 1970 afectó seriamente estas aspiraciones en lo relativo a la actividad política misma, pero no así en lo atinente a la diplomacia y la administración pública, campos donde los graduados pudieron insertarse. ${ }^{4} \mathrm{Tal}$ como sostienen Guiñazú y Gutiérrez, la Escuela de Ciencias Políticas “definió su perfil sin demasiadas contradicciones con el proceso político y social de aquellos años, extremando sus esfuerzos para formar "líderes políticos" que nutrieran a la nueva clase dirigente" (mimeo). En la Facultad de Ciencias Políticas y Sociales de la UNCuyo la carrera se modificó en respuesta a las necesidades de la dictadura militar que se propuso eliminar la ciencia Política, tal como lo hizo con la carrera de Sociología, dejando la Facultad dedicada a la formación de cuadros administrativos para la función pública. Primó en este período una perspectiva estrechamente tecnocrática (Sarlo 2007: 101) en la concepción de la ciencia. De esta forma se invirtió la denominación de la carrera que pasó a llamarse Licenciatura en Administración Pública y Ciencias Políticas y, prácticamente, se eliminaron los contenidos propios de la disciplina de la currícula.

Hasta principios de las década de 1980 las políticas educativas desarrolladas por los diversos gobiernos, orientadas a la formación y capacitación de cuadros políticos y administrativos se formularon e implementaron, principalmente, a través de las universidades y de fundaciones partidarias bajo la orientación político-ideológica de cada uno de ellas.

\subsection{La capacitación de los cuadros políticos en el retorno a la democracia}

La vuelta a la democracia en 1983 significó una nueva etapa para estas políticas. La imperiosa necesidad de consolidar el régimen democrático, de impulsar un proyecto de modernización del Estado y dar lugar a las crecientes demandas de participación política de la ciudadanía, plantearon nuevos dilemas para los cuales el Estado necesitaba contar con una clase dirigente capaz de "recuperar la política y recomponer el espacio público liberal como lugar de aparición de los sujetos de la democracia" (Quiroga 2005: 17). En este contexto, surge nuevamente el interrogante en torno a quiénes integrarían 
dicha clase dirigente y cuáles serían las instituciones a través de las cuales se podría articular su capacitación.

En el caso del gobierno de Raúl Alfonsín, la respuesta a estas cuestiones se pensó, por un lado, a través del acercamiento de intelectuales al gobierno y por el otro, a partir del proceso de modernización del Estado en democracia en el cual se establecían "canales de expresión adecuados a los partidos políticos, las organizaciones sociales, los municipios, las instituciones barriales y vecinales" (Alfonsín 1985). En este proyecto, a diferencia de lo que sucederá luego, no se explicitaba el rol de los partidos políticos, "el sistema de partidos parece ser el actor ausente de la propuesta modernizadora” (Quiroga 2005: 23). Más allá de lo cual, la capacitación política quedó, en este período, en manos de las fundaciones partidarias y de la Universidad de Buenos Aires (UBA), sin llegar a definirse, desde el Estado, una política específica que atendiera dicha demanda.

El partido en el gobierno contaba con instancias de formación, como la Fundación Karakachoff y la Fundación para el Cambio en Democracia (FUCADE), para el adoctrinamiento de la militancia partidaria. Mientras, el semillero de los cuadros políticos del radicalismo era la UBA y la Federación Universitaria Argentina (FUA). ${ }^{5}$

Con anterioridad a la caída del régimen militar, Alfonsín ya había mostrado su interés por acercar a los intelectuales a la política tras el desafio de "construir una nueva democracia" (Quiroga 2005: 23, 50). Esta convocatoria, destinada a intelectuales, independientes y afiliados del partido radical para participar en la elaboración de los textos presidenciales a través de los cuales se fijarían los temas centrales de la agenda política, permitió la configuración de una nueva cultura política democratizadora que iría a replantear las relaciones entre el intelectual y la política. Algunos de ellos, extrapartidarios que pertenecían a centros de investigación privados como el Centro de Investigaciones Sociales sobre el Estado y la Administración (CISEA) o el Centro de Estudios de Estado y Sociedad (CEDES) ocuparon puestos de primera importancia en los gabinetes de Alfonsín. Dante Caputo fue designado Ministro de Relaciones Exteriores y Jorge Sábato de Educación; Jorge Roulet fue nombrado Secretario de la Función Pública y Enrique Groisman Subsecretario de la Función Pública, mientras Oscar Oszlak ocupó la Subsecretaría de Investigación y Reforma Administrativa. Este grupo de intelectuales orgánicos, que nutrieron la clase política en el poder, ayudó a pensar teórica e ideológicamente el proceso de transición y consolidación democrática. Sumado a lo cual, el gobierno diseñó un espacio de discusión pluralista, representativo de los diferentes ámbitos en los que se organizó la vida colectiva, destinados a la producción de proyectos que se orientaran al logro de estos fines (Quiroga 2005: 46). De esta forma la necesidad de reestructuración del Estado, puso nuevamente de relieve la demanda de cuadros políticos formados para la generación de políticas públicas y técnicos capaces de gerenciarlas en el marco de una democracia participativa. 
En respuesta a esto último, en 1984, se crea el Cuerpo de Administradores Gubernamentales, siguiendo el modelo de la Escuela Nacional de Administración (ENA) de Francia. La estrategia del Gobierno era hacer frente de forma focalizada al problema de la función pública. Para ello se convocaría a las sucesivas promociones de Agentes Gubernamentales (AG) que integrarían las áreas críticas de Gobierno en la gestión de políticas públicas (Negri 2000). Se trataba de un programa destinado fundamentalmente a la formación de cuadros técnicos que trabajarían articuladamente con los cuadros políticos encargados de la formulación de políticas.

Sin embargo, hacia mediados de los años ochenta la fortaleza del proyecto del Gobierno radical comenzó a verse delimitada por la confrontación entre las estructuras partidarias y la lógica de formación y acumulación de poder de las corporaciones empresarias y sindicales, que irían a deteriorar los cimientos de la incipiente democracia participativa en pos de los propios intereses sectoriales. A partir de 1987 "la gravedad de la crisis, el poder de los centros financieros internacionales, y el peso de una sociedad altamente corporativa, doblegan la voluntad política del Gobierno, mientras el sistema de partidos se resiente y los ciudadanos pierden protagonismo y buscan desentenderse (en términos relativos) de la política" (Quiroga 2005: 24). En este contexto, en el que el gobierno no logró implementar muchas de las reformas propuestas, la recuperación de la eficiencia estatal no fue una preocupación central, con lo cual no se pudieron mejorar las capacidades estatales (Sidicaro 2001: 50).

\subsection{La creación de instituciones para la formación y capacitación política en el marco de la reforma constitucional}

En 1989 las situaciones hiperinflacionarias y anómicas llevaron al gobierno radical a adelantar el traspaso del poder al nuevo presidente electo, Carlos Menem. A partir de entonces y en el marco del agotamiento de un modelo de gestión pública populista o desarrollista (Cavarozzi 1996: 12), la reestructuración del aparato del Estado se impulsó a través de la implementación de un conjunto de reformas - de primera y segunda generación- siguiendo las recetas de organismos de financiamiento internacional. En el marco de dichos esquemas modernizadores del Estado volvió a surgir una problemática tradicional del sector público: el desdibujamiento en la frontera entre política y administración. La crisis del Estado intervencionista fue el contexto propicio para que volviera a plantearse la necesidad de que, desde el Estado, se aliente la formación de liderazgos políticos, administrativos y sociales acordes a los procesos de modernización. En este sentido, la carencia de espacios de interacción diferenciados entre las estructuras administrativas y las políticas, e inclusive entre estas últimas y los partidos políticos, constituyó uno de los principales problemas a resolver, más allá de la necesaria complementariedad funcional que un modelo de gestión pública eficaz y eficiente requiere de los mismos (CLAD 1997: 6). 
En este escenario que evidencia la indefinición en la delimitación de los espacios que corresponden a la política y a la administración, donde conviven funcionarios administrativos deslegitimados en su función, técnicos apartidarios, políticos-gerentes y lógicas partidocráticas que impiden la conformación de aparatos estatales eficientes al mando de una conducción política capaz de dar respuesta a las demandas de la sociedad (Thwaites Rey 2005:105), se implementó a mediados de los años noventa una política orientada a la formación y capacitación de cuadros políticos. A través de la misma se intentó responder al problema de "la limitada influencia que los institutos y escuelas de administración han tenido en la capacitación de liderazgo político de sus respectivos países y, como consecuencia, el restringido peso de las mismas en la conducción de los destinos del Estado y la sociedad" (CLAD 1992: 6). De esta manera la formación de los políticos volvió a ser una cuestión de agenda de los organismos estatales dedicados a la capacitación de funcionarios públicos.

En el marco de la reforma constitucional de 1994, esta problemática vuelve a adquirir rango constitucional al reconocerse a los partidos políticos como instituciones fundamentales del sistema democrático y, en consecuencia, consagrar la capacitación de cuadros políticos en tanto "política de Estado orientada a la gestión del sector público y liderada por el Estado” (ENG 1998:9). De esta manera, se crearon dos instancias de formación y capacitación político estatales, una en el ámbito de la Secretaría de la Función Pública: la Escuela Nacional de Gobierno -ENG- dependiente del Instituto Nacional de Administración Pública -INAP-, organismo rector de la capacitación del Estado argentino; y la otra en la órbita de la Subsecretaría de la Juventud del Ministerio del Interior: el Instituto de Capacitación y Formación de Dirigentes Políticos.

El Instituto de Capacitación y Formación de Dirigentes Políticos, con dependencia funcional del Subsecretario de la Juventud del Ministerio del Interior comenzó a funcionar en el año 1995 por Resolución 774/95. Su finalidad era la de organizar "cursos y seminarios en el campo del derecho, la política, la economía y las relaciones internacionales"; realizar "estudios e investigaciones en las áreas mencionadas" y "propiciar el contacto directo con la clase dirigente de aquellos que se inscriben en los cursos y seminarios" (Art. $3^{\circ}$ ). De esta forma, se buscaba responder a los "cambios en la dinámica de las funciones de gobierno" producidos por la reforma constitucional que impactarían en el "diseño de un nuevo Estado", que "exigirá una capacitación especializada de la dirigencia política argentina... a fin de operativizar eficientemente las acciones políticas" (Considerando, Res. 1664 del Ministerio del Interior).

Ese mismo año inició la implementación del Programa Escuela Nacional de Gobierno en el ámbito del Instituto Nacional de Administración Pública (INAP). Su finalidad, definida por Resolución 379 de la Secretaría de la Función Pública, era la de "formar y 
capacitar a dirigentes políticos” (Art. $1^{\circ}$ ). La normativa preveía que el programa estaría dirigido por un Consejo Rector presidido por el Directorio del INAP y conformado por representantes de los partidos políticos legalmente constituidos y con representación parlamentaria no menor a cuatro bancas, así como también invitaba al Ministerio del Interior y al Consejo Interuniversitario Nacional (CIN) a proponer un representante para que formara parte del mismo (Art. $3^{\circ}$ ). Dentro de las atribuciones del Consejo Rector se encontraban: la de "proponer, coordinar y supervisar las condiciones de ingreso de los aspirantes a la Escuela”; “elaborar el diseño curricular para la capacitación de los dirigentes políticos en los poderes legislativos y ejecutivo de los distintos niveles de Gobierno; "elevar la propuesta de designación del plantel docente"; "diseñar un régimen de pasantías"; "aprobar el reglamento de funcionamiento interno de la Escuela Nacional de Gobierno"; "proponer programas y convenios de cooperación técnica externa e interna para cumplir con los cometidos de la Escuela" y "proponer un programa de becas para aspirantes a ingresar a la Escuela Nacional de Gobierno” (Art. $4^{\circ}$ ).

A través de estas instituciones, que desde su creación se superpusieron en relación a sus objetivos y finalidades sin llegar a articularse y menos aún a unificarse, el Estado buscaba dar respuesta al mandato constitucional a través de la formación y capacitación de una clase política apta para la gestión y conducción del aparato estatal.

La política desarrollada se fundamentó en el principio de que al tratarse de una cuestión que involucra valores éticos y morales es el mismo Estado el que debe hacerse cargo de la formación y capacitación de su clase política. "La formación de la dirigencia política es algo demasiado valioso como para dejarlo librado a la voluntad de los partidos políticos. La Constitución obliga al Estado a participar en la formación de la dirigencia política a los efectos de mejorar y profundizar la democracia e igualar las oportunidades de los argentinos para su participación y actuación política” (ENG 1998: 6). Desde esta visión, la formación de cuadros políticos a cargo de instituciones estatales se basó en objetivos muy diferentes a los encarados tradicionalmente por los partidos políticos, principalmente la ambiciosa política implementada -en términos políticos, académicos y presupuestarios- por la Escuela Nacional de Gobierno.

Sin embargo, de acuerdo a lo dispuesto por el artículo 38 de la CN y de la fundamentación que los constitucionales dieron al mismo, el Estado se comprometía a contribuir al sostenimiento económico de las actividades de los partidos políticos y de la capacitación de sus dirigentes, con lo cual su obligación consistía en garantizar el financiamiento de dicha actividad y controlar el cumplimiento de la misma, lo que dista bastante de implementar desde el Estado una política de formación y capacitación de cuadros políticos. De hecho, la incorporación al artículo 38 propuesta por el convencional constituyente por el Partido Justicialista de Córdoba, el Dr. Juan Carlos Maqueda, referida a la formación y capacitación de los dirigentes de los partidos políticos, ${ }^{6}$ se apoyó en la convicción 
de que "si bien es cierto que alguien puede sostener que esto es producto de la ley o, valga la redundancia, que dentro de la expresión sostenimiento económico está incluido todo, quiero que quede claro que una cosa son los fondos que destina el Estado legítimamente para la organización y el funcionamiento de los partidos políticos, y otra cosa son los fondos que destina para la capacitación y formación de la dirigencia política. De la misma forma en que el Estado sostiene económicamente el funcionamiento de los partidos políticos y puede exigirles publicidad sobre el origen y destino de los fondos, podrá controlar que sean destinados a la capacitación y formación de dirigentes y no se desvíen de sus fines específicos o sean utilizados para lo que puede representar una tentación permanente; la maquinaria electoralista del partido" (Maqueda 1994: 18291938). La intención de Maqueda era crear, a partir del reconocimiento constitucional, instituciones para la capacitación y la formación de los dirigentes, que pertenecieran a los partidos políticos y que fueran financiadas por el Estado. "Tenía en mente lo que son las famosas fundaciones alemanas, la Fundación Konrad Adenauer, que es la más conocida, la Friedrich Ebert, o sea, todas las fundaciones que tiene cada partido político alemán (Entrevista: Maqueda 2012).

Es decir que, a diferencia de lo dispuesto por el artículo 37/CN 1949, donde el Estado encargaba a las universidades la formación de dirigentes políticos, el artículo 38/CN 1994 delega dicha responsabilidad al sistema de partidos bajo el financiamiento y control del Estado. Ambos artículos tienen un sentido distinto, mientras el artículo 38 de la Constitución Nacional de 1994 responde a un interés central -el reconocimiento de los partidos políticos- y como derivación se plantea la capacitación de cuadros políticos, el artículo 37 de la Constitución Nacional de 1949 respondía directamente a la necesidad de formar y capacitar clase dirigente. En este sentido, es confusa la forma en la que se interpretó la disposición constitucional de 1994, no dejando en claro quién y cómo debería afrontar los procesos de formación y capacitación de la dirigencia política.

En el año 1995 los funcionarios involucrados y los representantes de los partidos políticos con representación parlamentaria interesados en la misma recurrieron, para la implementación de la política, a la experiencia en la capacitación de funcionarios públicos del INAP en la órbita de la Secretaría de la Función Pública y de otros programas implementados en el ámbito del Ministerio del Interior; a experiencias de capacitación política desarrolladas en el pasado, que más allá de sus resultados no tuvieron continuidad en el tiempo; a modelos internacionales como el de la Escuela Nacional de Administración de Francia (ENA), el de las Fundaciones Políticas alemanas y la Escuela de Gobierno John F. Kennedy de la Universidad de Harvard; a recomendaciones desde el ámbito académico; y al logro de consensos entre los principales partidos políticos en torno a los contenidos y docentes de los programas.

En este período, la idea de aprobar la creación de dichas instancias de formación y capacitación política a través de una ley que garantizara la continuidad institucional de las mismas estuvo presente pero no llegó a concretarse. El proyecto presentado en el año 
1999 por Leopoldo Moreau y Antonio Cafiero para la creación por ley de la Escuela Nacional de Gobierno así lo demuestra.

\subsection{La formación y capacitación política en un contexto de crisis}

Hacia fines de los años noventa los programas de formación y capacitación de cuadros dirigentes no fueron ajenos a un contexto en el cual la política había perdido centralidad en la relación Estado/Sociedad, vaciándose de contenidos y siendo reemplazada, en muchos casos, por el tecnicismo imperante en la época.

Más allá de la continuidad institucional de los programas de formación y capacitación política, en 1999, con el gobierno de la Alianza, disminuyó la importancia otorgada a la función pública. En un contexto generalizado de reducción presupuestaria y de cargos políticos, se desjerarquizó el área pasando de Secretaría a Subsecretaría. En consecuencia, la Escuela Nacional de Gobierno, con el cambio de gestión, abandonó el modelo de formación que había sido implementado desde su creación en el año 1995 poniendo en práctica uno mucho más orientado a la lógica academicista, siguiendo la impronta del modelo norteamericano y el de la ENA en Francia. Mientras el INCaP, que cambió su nombre por el de Instituto Nacional de Capacitación Política bajo la órbita de la Subsecretaría de Asuntos Institucionales y Relaciones con la Comunidad del Ministerio del Interior, continuó, sin mayores modificaciones, orientando sus actividades a la formación de "cuadros políticos partidarios" (La Nación 2 de julio 2000). En ambas instituciones se redujeron las horas cátedra y los recursos disponibles; la formación y capacitación política estaba siendo relegada a un segundo plano. Finalmente, tras la violenta crisis institucional, política, económica y social del año 2001 y la renuncia del presidente Fernando De la Rúa, los cursos anuales de formación y capacitación política implementados fueron abruptamente interrumpidos, aunque se continuaron desarrollaron algunas actividades.

La crisis de 2001 reveló las debilidades de una clase política más abocada a sostenerse electoralmente que a construir un proyecto político consensuado o, en principio, concertado. Una clase política integrada a una maquinaria electoral y de poder que ella misma contribuyó a consolidar, y a la que "le faltó una lectura de los signos de los tiempos y manifestó una falta de capacidad para construir agenda propia, para gobernar más que para administrar” (García Delgado 2003: 45).

En este contexto de emergencia económica y social, a principios del 2002, Eduardo Duhalde fue elegido Presidente de la Nación por la Asamblea Legislativa en aplicación de la Ley de Acefalia. Comenzó un nuevo período donde las prioridades del gobierno se redireccionaron fundamentalmente a la provisión de bienes y servicios públicos básicos como la salud, la educación, y los programas de promoción y seguridad social (Memoria del Estado de la Nación Año 2002. 2003: 712). 
En medio del desencanto y descreimiento en la política, en los dirigentes y en las instituciones, el presidente Duhalde convocó a un proceso abierto de diálogo apoyándose en la iniciativa que con anterioridad a la renuncia de De la Rúa venía promoviendo el Episcopado Argentino apoyado por el Programa de las Naciones Unidas para el Desarrollo (PNUD), con el objeto de conformar la mesa del Diálogo Argentino. A través de este proyecto se buscaba "recuperar la confianza de los argentinos en sí mismos y relanzar todo el potencial del país” (Diálogo Argentino, 2002:1). Para ello se congregó a los distintos sectores sociales, incluidos los partidos políticos, empresas, sindicatos, bancos, organizaciones no gubernamentales, sociales y de la ciencia y de la cultura, las asociaciones de consumidores, los sectores emergentes, diversas personalidades de la Argentina y a la ciudadanía en general. En la búsqueda por alcanzar amplios consensos entre la clase dirigente, la ENG a través de su experiencia en la formación y capacitación de cuadros políticos cooperó brindando soporte académico al proyecto del Diálogo Argentino (ENG, 2002: 8). De esta forma, la capacitación de dirigentes implementada a través del Programa ENG debía dar respuesta a una serie de desafios propios de la situación que vivía el país, que permitiera vislumbrar a futuro las profundas transformaciones demandadas por la sociedad (Bellagio 2002: 1,2). Algo bastante dificil de lograr en un contexto en el cual, la crisis de representación política junto al deterioro en el nivel académico, venían transformando a la Escuela en parte del sistema de clientelismo de los partidos políticos.

Por otro lado, el INCaP, frente a la carencia de recursos, instrumentó un modelo de gestión asociada principalmente con las organizaciones no gubernamentales y distintos sectores de la sociedad civil. El desafio era acercar nuevamente la sociedad a la política recreando los ámbitos de participación social. Para ello, el Instituto orientó sus actividades a las demandas de los nuevos actores sociales que emergían en el escenario posterior a la crisis del 2001. De esta forma, los programas de formación y capacitación política se llevaron a cabo a través de cursos, talleres, seminarios y jornadas a demanda de los interesados, redefiniendo el perfil de los destinatarios que dichas políticas habían tenido en los años noventa. Lo mismo sucedió con los docentes y los contenidos temáticos, que pasaron a delinearse a propuesta de las organizaciones involucradas en cada instancia de capacitación.

Ni la gravedad de la crisis presupuestaria o el objetivo común e imperioso por dar respuesta a las demandas sociales permitieron que ambas instancias de formación encontraran espacios de articulación o unificación. La ENG trató de mantener la estructura operativa inicialmente planteada, a través de un Consejo Consultivo de carácter político y de composición plurisectorial, y un Consejo Académico de composición plural desde lo ideológico, incorporando las visiones de diferentes sectores y actores sociales orientadas a definir cómo debía plantearse la formación y capacitación de dirigentes políticos y sociales en el marco de la reforma política. El INCaP, por su parte, territorializó la gestión orientando sus acciones al ámbito local y al militante de base. 


\subsection{La capacitación política se orienta a la formación ciudadana}

Con la asunción de Néstor Kirchner a la presidencia de la República comenzó un proceso, tal como se lo denominó desde el propio gobierno, de "reconstrucción nacional bajo las premisas de la emergente cuestión social, la rearticulación entre política y sociedad, y las cuestiones vinculadas a la transparencia estatal". La crisis fue el contexto propicio a partir del cual surgió un debate cuyo eje central fue la necesidad de iniciar un proceso de ingeniería institucional. El proceso de Reforma Política se orientó de esta forma a fortalecer institucionalmente al Estado para que el mismo se legitimara haciendo frente a las necesidades y exigencias de la sociedad (Memoria del Estado de la Nación Año 2003. 2004: 199). Una sociedad en la que prevalecía "una sensación reiterada de incumplimiento de promesas y de falta de voluntad y de abroquelamiento de la dirigencia política detrás de los recursos de poder de que dispone para digitar la nominación de los representantes" (Mora y Araujo 2005: 133). En este sentido y más allá de que la capacitación de cuadros políticos que permite la renovación dirigencial constituye solamente uno de los aspectos en la recuperación de la legitimidad del sistema democrático representativo, volvía a ser un tema de agenda en el ámbito de la Secretaría de la Función Pública, pero principalmente en el del Ministerio del Interior.

Se trata de un período en el que las instituciones estatales orientadas a la formación y capacitación política, a pesar de algunas acotadas actividades realizadas en conjunto, definen diversos rumbos de acción, en parte debido al apoyo político y a los recursos con los que cada una contó para diseñar y ejecutar los programas de capacitación política. Más allá de lo cual, un rasgo común a ambas gestiones, fue el alejamiento de las universidades por un lado y de la prácticamente nula articulación con los partidos por el otro.

En el caso de la ENG, teniendo en cuenta que Gestión Pública volvió al rango de Secretaría en la órbita de la Jefatura de Gabinete, podría haberse esperado un mayor apoyo político a dicho Programa. Sin embargo, no hubo una clara decisión política al respecto. Los partidos políticos tampoco se mostraron preocupados por alentar instancias de formación y menos aún acudir a las convocatorias realizadas desde los aparatos estatales por considerarlos formas de coaptación del partido gobernante, lo que terminó influyendo negativamente en el desarrollo del Programa.

Sin apoyo político y sin demasiados recursos, la ENG redefinió una vez más su perfil. Orientada a la formación de dirigentes políticos y sociales y a la construcción de un espacio de encuentro entre la dirigencia política tradicional y los nuevos actores de la sociedad, como los movimientos sociales, se dictaron seminarios de carácter trimestral específicos en diversas temáticas -Gobierno y Sociedad; análisis de políticas públicas; historia; economía; técnica presupuestaria y legislativa; sistemas electorales; administración financiera; etc.- y charlas abiertas al público en general. No obstante, al no contar con un presupuesto propio, el Programa no llegó a realizarse por fuera de la Ciudad 
de Buenos Aires, como se había previsto. Por el contrario y como parte de la reforma política impulsada desde el Ministerio del Interior, se asignaron nuevos recursos para el desarrollo de la política orientada a la capacitación de dirigentes políticos y sociales. En este contexto, el INCaP pasó a la órbita de la Subsecretaría de Asuntos Políticos y Electorales incorporando un nuevo programa de capacitación.

La nueva versión del Instituto fue creada con el objetivo de "alcanzar a través de políticas activas de capacitación, encuadradas dentro de un marco académico institucionalizado, la cualificación de los ciudadanos, como sujetos portadores de valor político, a fin de aportar al mejoramiento de la calidad político-institucional de la Nación" (Resolución 452/04). La intención era recuperar la política como herramienta para el cambio y la capacitación política de la ciudadanía era parte de este objetivo.

El Instituto, que contaba con una partida presupuestaria específica, amplió el perfil de sus destinatarios y definió, a partir de diversos trayectos formativos, un itinerario secuencializado de módulos que les permitía a los cursantes abarcar áreas de conocimiento integradas. Además, se implementó una fuerte política de federalización de la capacitación política a través de convenios con provincias y municipios del Gran Buenos Aires y del interior del país, principalmente con los que respondían al partido al frente del gobierno nacional y también con fundaciones, organizaciones de la sociedad civil y sindicatos.

\subsection{La política estatal de formación y capacitación política en el proyecto nacional y popular}

Cristina Fernández de Kirchner asumió la presidencia de la Nación en diciembre de 2007. Su período de gobierno es el que quizás más contrastes presenta con la gestión de Carlos Menem, donde la política se encontraba fuertemente desvalorizada. En el año 2008 las condiciones de desarrollo de la política y los conflictos que enfrentados por el gobierno, como la discusión de la Resolución $125^{7}$ y la aprobación de la "ley de medios" le generaron la necesidad de fortalecer la construcción de un "relato kirchnerista", que ya había comenzado durante la gestión de Néstor Kirchner, vinculado a la revalorización de la política en el imaginario público.

La política es ahora impulsada como herramienta del cambio y la transformación. Las acciones del gobierno se orientan a la "construcción de un proyecto nacional más equitativo y plural, como así también hacia el fortalecimiento del sistema democrático en pos de su consolidación" (INCaP 2011: 4). Consecuentemente, las políticas destinadas a la formación y capacitación política se reformulan en función de la orientación del gobierno. Se trata de políticas gubernamentales que responden a necesidades de coyuntura. "Nuestro objetivo general es formar dirigentes que incrementen sus capacidades para identificarse con el proceso político en marcha, reflexionen con sentido crítico 
sobre los elementos teóricos del Modelo Nacional y Popular y puedan volcarlos a la práctica política concreta en su territorio" (INCaP, 2011).

En el 2007 y más allá de la continuidad del mismo partido en el gobierno, cambió nuevamente la dirección del INCaP. Tras las modificaciones producidas en la institución durante la gestión anterior, se implementó un nuevo programa de formación y capacitación, el Programa Nacional de Formación de Dirigentes y Fortalecimiento Institucional, con el objeto de "fomentar el federalismo a través de la participación a nivel nacional de los distintos estamentos de la sociedad en las actividades de capacitación, realizando cursos y seminarios en forma coordinada y conjunta con gobiernos provinciales y organizaciones no gubernamentales" (Memoria del Estado de la Nación Año 2011, 2012: 38).

La ENG, por su parte, y luego de la gestión anterior en la que contó con escasos recursos y apoyo político, a partir de la asunción del nuevo gobierno en 2007 dejó de funcionar hasta fines de 2010, cuando se designó a un nuevo director. La nueva gestión cambió radicalmente los fundamentos bajo los cuales se creó la Escuela en 1995, para implementar un programa basado en el uso de las nuevas tecnologías en materia de estrategias políticas. Con dicho objetivo y bajo la lógica del "Modelo nacional y popular", cambiaron también los destinatarios de la política, que pasaron a ser desde adolescentes con algún tipo de militancia barrial hasta adultos mayores del PAMI que se acercaban a la Escuela para acceder a cursos sobre el uso de internet.

\section{La gubernamentalización de la política y la inestabilidad del modelo de formación y capacitación de cuadros políticos}

Más allá del devenir de estas políticas, lo cierto es que desde el primer gobierno de Juan Domingo Perón, tanto en los períodos de dictaduras militares como en los de los gobiernos constitucionales, existieron iniciativas en distinto grado de desarrollo que partían de la necesidad de formar y capacitar dirigencia política y cuadros técnicos para la gestión pública.

Sin embargo, en el caso de las instituciones estatales creadas a mediados de la década de 1990, destinadas a la formación y capacitación de dirigentes políticos fueron cerrándose cada vez más a las necesidades coyunturales de cada gestión, generando la gubernamentalización de componentes estatales de la gestión, en torno a lo cual sigue sin quedar claro ¿en qué tienen que formar y capacitar dichas instituciones? y ¿A quién tienen que formar? Las respuestas a estas interrogantes no han sido lo suficientemente precisas. Es por ello que la política de formación y capacitación de cuadros políticos ha estado muy ligada a cuestiones coyunturales de los gobiernos y no al Estado. Aunque la cuestión aparezca recurrentemente ligada a los proyectos de reforma del Estado y a los constantes 
intentos de reconstrucción de la política argentina, siempre terminó diseñada en respuesta a los requerimientos del gobierno y no a lo que necesitan el Estado, el sistema de partidos y la sociedad. La idea de la "Argentina en emergencia permanente" (Quiroga 2005) ilustra los diversos contextos de crisis en los que la demanda de cuadros políticos vuelve a instalarse en el Estado y la Sociedad, aún sin poder resolverse, careciendo la Argentina de una clase dirigente formada y capacitada para la gestión de los asuntos estatales.

Es por ello que hasta el momento dicha necesidad no termina de institucionalizarse más allá de las iniciativas particulares alentadas por cada gobierno. Los paradigmas teóricos desde donde se ha pretendido formular e implementar esta política han sido diversos y en consecuencia el quién lo hace y el cómo se hace ha tenido en cada gestión respuestas diferentes. Se trata de un proceso inconcluso, en el que los partidos políticos también fracasaron como responsables de esta tarea. "Histórica y genéricamente, los partidos no se han propuesto sistemáticamente mejorar la calidad de los cuadros, con nefastas consecuencias para el sector público en particular, y para la sociedad en general" (Thwaites Rey 2005: 111). Mientras a las universidades tampoco se les otorgó el rol explícito de formar y capacitar a la dirigencia política, aunque siempre tuvieron iniciativas al respecto.

Se trata de una política que ha sido formulada con la intención de adquirir el rango de una política de Estado, aunque en los hechos ha quedado atrapada en la lógica de la particular ideología del oficialismo gubernamental. Este es el caso de la política implementada a través de la ENG y del INCaP, que más allá de su continuidad temporal, son aparatos estatales destinados a la formación política cada vez más cerradas a la visión que cada gestión tiene de los procesos de capacitación de la dirigencia política. De esta forma, agencias estatales creadas para la formación de los cuadros políticos del Estado se convierten en órganos de gobierno y la política adquiere el sesgo partidario, desde donde se produce "una captura de lo público en función de intereses que no necesariamente son colectivos" (Negri 2000: 8), relegando los intereses de la sociedad. Sumada a ello, la indefinición de una política de Estado orientada a la capacitación de la dirigencia política no ayuda en la delimitación de los espacios entre cuadros administrativos, cuadros políticos, y tecnócratas. "La falta de idoneidad para conducir con solvencia segmentos especializados del Estado por parte de los funcionarios políticos, puede derivar en problemas serios de articulación con la llamada "línea" de funcionarios técnicos - permanentes o contratados - que son los encargados de llevar a la práctica los lineamientos trazados, y así hacer naufragar las propuestas mejor intencionadas" (Thwaites Rey 2005: 111)

En definitiva, la política de formación y capacitación de cuadros políticos implementada por la ENG y el INCaP ha estado ligada a coyunturas políticas de los gobiernos y no al Estado. Esto es así porque, por un lado, el Estado no ha tenido la claridad conceptual ne- 
cesaria para entender la naturaleza del problema y ha tratado de resolverlo trasladándole la responsabilidad a los partidos políticos o creando agencias estatales que quedan atrapadas en la particular ideología del oficialismo gubernamental. Por el otro lado, los partidos no han utilizado el financiamiento del Estado para la capacitación de sus cuadros y tampoco cuentan con los mecanismos para hacerlo, consecuentemente acceden al poder sin contar, muchas veces, con dirigentes políticos formados. Estas experiencias han tenido resultados e impactos diversos, que nos demuestran que en Argentina no se logrado aún estabilizar un modelo para la formación y capacitación de los cuadros políticos.

\footnotetext{
${ }^{1}$ El presente trabajo es parte de la tesis doctoral “¿El Estado sin políticos? La formación y capacitación política en la Argentina democrática”. Presentada y aprobada en la Escuela de Gobierno de la UNSAM en el mes de Abril de 2013.

2 "Los partidos políticos son instituciones fundamentales del sistema democrático. Su creación y ejercicio de sus actividades son libres dentro del respeto a esta Constitución, que garantiza su organización y funcionamiento democrático, la representación de las minorías, la competencia para la postulación de candidatos a cargos públicos electivos, el acceso a la información pública y la difusión de sus ideas. El estado contribuye al sostenimiento económico de sus actividades y de la capacitación de sus dirigentes. Los partidos políticos deberán dar publicidad del origen y destino de sus fondos y patrimonio" (Artículo 38 de la Constitución Nacional).

3 “Las Universidades establecerán cursos obligatorios y comunes destinados a los estudiantes de todas las facultades para su formación política, con el propósito de que cada alumno conozca la esencia de lo argentino, la realidad espiritual, económica, social y política de su país, la evolución y la misión histórica de la República Argentina, y para que adquiera conciencia de la responsabilidad que debe asumir en la empresa de lograr y afianzar los fines reconocidos y fijados por esta Constitución" (Constitución 1949. Capítulo III, art. 37, sección IV, inciso 4).

${ }^{4}$ Información extraída de la página de la UCA disponible en http//:www.uca.edu.ar (Recuperado 17 de marzo, 2011)

${ }^{5}$ De hecho, este contexto fue favorable para la postergada creación de la carrera en ciencias políticas en la Universidad de Buenos Aires (UBA). En 1986 la apertura de la carrera "intentó, por un lado, concentrar la actividad académica que se encontraba tan dispersa en centros de investigación no universitarios, y por otro, la formación de una masa intelectual democrática e influyente en la dirección de la política a través de la transmisión del conocimiento científico de la politica" (Bulcourf y D'Alessandro, 2003: 162).

6 "El Estado contribuye al sostenimiento económico y la capacitación y formación de los dirigentes de los partidos políticos, quienes deberán dar publicidad del origen y destino de sus fondos y patrimonios" (Disidencia propuesta por el Dr. Juan Carlos Maqueda. Debate del dictamen de la Comisión de Redacción en los despachos en mayoría y minoría originados en la Comisión de Participación Democrática. 1994: 1829-1938). ${ }^{7}$ A través de la Resolución 125/08 el gobierno intentó incrementar las retenciones a las exportaciones de soja y girasol y establecer un sistema móvil para estas. En respuesta a esta medida, el sector agropecuario se manifestó en contra provocando un paro generalizado y el bloqueo de rutas. Mientras el proceso continuó politizándose y otros sectores políticos, económicos y sociales comenzaron también a manifestar sus posturas. En el mes de junio, el proyecto de ley por las retenciones fue enviado para su tratamiento al Congreso de la Nación, siendo finalmente rechazado por el Poder Legislativo y posteriormente dejado sin efecto por el Poder Ejecutivo.
} 


\section{ANEXO}

CUADRO 1. Matriz teórico-analítica para el estudio de la política estatal orientada a la formación y capacitación de cuadros políticos ${ }^{1}$

\begin{tabular}{|c|c|c|}
\hline $\begin{array}{l}\text { Política estatal } \\
\text { Componentes }\end{array}$ & $\begin{array}{l}\text { Estado como relación } \\
\text { social }\end{array}$ & $\begin{array}{l}\text { Estado como conjunto de } \\
\text { instituciones }^{2}\end{array}$ \\
\hline $\begin{array}{l}\text { Momento histórico en el que } \\
\text { por primera vez se explicita, } \\
\text { desde el Estado, la necesidad } \\
\text { de contar con una política de } \\
\text { formación y capacitación para } \\
\text { los cuadros políticos }\end{array}$ & $\begin{array}{l}\text { Ideas que justifican la } \\
\text { problematización del asunto }\end{array}$ & $\begin{array}{l}\text { Instituciones a través de las cuales } \\
\text { se problematiza el asunto }\end{array}$ \\
\hline $\begin{array}{l}\text { Contexto político, económico, } \\
\text { social e ideológico en el que la } \\
\text { formación de cuadros políticos } \\
\text { forma parte del conjunto de } \\
\text { problemas sociales que requieren } \\
\text { solución }\end{array}$ & $\begin{array}{l}\text { Contexto de las ideas en el } \\
\text { que la formación de cuadros } \\
\text { políticos constituye un } \\
\text { problema social }\end{array}$ & $\begin{array}{l}\text { Contexto institucional en el que } \\
\text { la formación de cuadros políticos } \\
\text { se plantea como un problema } \\
\text { social }\end{array}$ \\
\hline $\begin{array}{l}\text { Definición del problema a } \\
\text { solucionar }\end{array}$ & $\begin{array}{l}\text { Definición del problema / } \\
\text { Perfil de los destinatarios / } \\
\text { Ideas que permiten al Estado } \\
\text { la formación de consenso en } \\
\text { la sociedad }\end{array}$ & $\begin{array}{l}\text { Forma en que se plantea la } \\
\text { relación entre la lógica política y } \\
\text { la lógica administrativa/técnica }\end{array}$ \\
\hline $\begin{array}{l}\text { Toma de posición del Estado: } \\
\text { política estatal }\end{array}$ & $\begin{array}{l}\text { Tipo de política: de Estado o } \\
\text { de gobierno / Ideas que dan } \\
\text { fundamento a la política } \\
\text { Finalidad de la política }\end{array}$ & $\begin{array}{l}\text { Niveles de institucionalidad } \\
\text { de la política / Definición de } \\
\text { contenidos específicos }\end{array}$ \\
\hline $\begin{array}{l}\text { Actores que han participado en } \\
\text { la resolución del problema }\end{array}$ & $\begin{array}{l}\text { Formas en la que cada actor } \\
\text { ha fundamentado la necesidad } \\
\text { de resolución del problema y } \\
\text { su participación en la misma }\end{array}$ & $\begin{array}{l}\text { Instituciones que los actores } \\
\text { utilizan para la resolución del } \\
\text { problema }\end{array}$ \\
\hline $\begin{array}{l}\text { Prácticas de los actores } \\
\text { involucrados en la resolución del } \\
\text { problema }\end{array}$ & $\begin{array}{l}\text { Ideas sobre las cuales se } \\
\text { fundamentan las prácticas de } \\
\text { los actores }\end{array}$ & $\begin{array}{l}\text { Prácticas de los actores } \\
\text { Instituciones dentro de las cuales } \\
\text { los actores realizan sus prácticas }\end{array}$ \\
\hline $\begin{array}{l}\text { Recursos y apoyos que los } \\
\text { actores movilizan para la } \\
\text { resolución del problema }\end{array}$ & $\begin{array}{l}\text { Consenso o recurso de } \\
\text { confianza y de mayoría }\end{array}$ & $\begin{array}{l}\text { Recurso jurídico; humanos; } \\
\text { económicos; cognitivos; } \\
\text { relacionales; cronológico y } \\
\text { patrimoniales }\end{array}$ \\
\hline Cristalizaciones institucionales & $\begin{array}{l}\text { Ideas que dan sustento a la } \\
\text { creación o modificación de } \\
\text { las instituciones }\end{array}$ & $\begin{array}{l}\text { Instituciones a través de las cuales } \\
\text { se implementa la política }\end{array}$ \\
\hline $\begin{array}{l}\text { Modo de resolución del } \\
\text { problema }\end{array}$ & $\begin{array}{l}\text { Fundamentación del modo de } \\
\text { resolución del problema }\end{array}$ & $\begin{array}{l}\text { Instituciones a través de las } \\
\text { cuales se ha decidido, de forma } \\
\text { dominante, resolver el problema } \\
\text { en cada período }\end{array}$ \\
\hline $\begin{array}{l}\text { Cambios sociales e internos al } \\
\text { Estado atribuibles a la política }\end{array}$ & $\begin{array}{l}\text { Impacto de la política en el } \\
\text { Estado / Valores tendientes } \\
\text { al fortalecimiento de la } \\
\text { democracia / Modificaciones } \\
\text { en la relación entre } \\
\text { gobernantes y gobernados }\end{array}$ & Cambios institucionales \\
\hline
\end{tabular}

${ }^{1}$ En el trabajo de tesis doctoral se incorporan a la Matriz los indicadores que permitieron el análisis de cada uno de los componentes de la política.

${ }^{2}$ Mabel Thwaites Rey se refiere a esta dimensión del Estado como conjunto de aparatos (2005). 


\section{Referencias Bibliográficas}

Abal Medina, Juan y Suarez Cao, Julieta (2002) "La competencia partidaria en la Argentina: sus implicancias sobre el régimen democrático". En Cavarozzi, Marcelo y Abal Medina, Juan: El asedio a la política. Los partidos latinoamericanos en la era neoliberal. Santa Fe: Homo Sapiens, 2002: 163-186.

Bulcourf, Pablo A. y D’Alessandro, Martín (2003) "La Ciencia Política en la Argentina”. En Pinto, Julio. Introducción a la Ciencia Política. Buenos Aires. Ed. Universitaria de Buenos Aires, 2003: 133-184.

Cueto, Walter y Guardamagna, María Melina (2011): "Políticas de Estado en democracia". Revista El Estadista. 39: 20-21.

Cueto, Walter y Guardamagna, María Melina (2012) “¿Hay políticas de Estado en la Argentina? Aproximaciones a un concepto". Documentos y Aportes en Administración Pública y Gestión Estatal (DAAPGE). Facultad de Ciencias Económicas. Universidad Nacional del Litoral, Argentina, 18: 7-26.

De Imaz, José Luis (1964): Los que mandan. Buenos Aires. Editorial Universitaria de Buenos Aires.

Dugini, María Inés y Lopez, Marta Iris (1995): Breve Reseña Histórica de la Creación de Nuestra Facultad. Facultad de Ciencias Políticas y Sociales. Universidad Nacional de Cuyo. Mendoza.

García Delgado, Daniel (2003): Estado-nación y la crisis del modelo. Buenos Aires: Grupo Editorial Norma.

Guardamagna, María Melina (2011): “Marchas y contramarchas en el desarrollo de la ciencia política argentina”. Persona y Sociedad. Volumen XXV (3):11-27.

Guardamagna, María Melina (2012): "Una política de Estado para la función pública? Aciertos y desaciertos en la formación y capacitación de cuadros políticos en democracia". Trabajo y Sociedad, 19: 451-464.

Guardamagna, María Melina (2013): “El rol de la universidad en la formación de cuadros políticos en la Constitución de 1949”. POSTdata 18 (1):73-102.

Guiñazú, María C. y Gutiérrez, María A. "La Ciencia Política en Argentina: de la inestabilidad a la transición". Mimeo. 
Halperin Donghi, Tulio (2008): Son memorias. Buenos Aires: Siglo XXI Editores.

Matus, Carlos (2008) El líder sin estado mayor: la oficina del gobernante. San Justo, Pcia. de Buenos Aires: Universidad Nacional de la Matanza.

Negri Sergio Ernesto (2000): "La Función Pública en la República Argentina: Impresiones de una historia reciente de avances y retrocesos. El caso del Cuerpo de Administradores Gubernamentales”. Trabajo presentado al V Congreso Internacional del CLAD sobre la Reforma del Estado y la Administración Pública. Octubre.

Oszlak, Oscar y O’Donnell, Guillermo (1976): "Estado y políticas estatales en América Latina: hacia una estrategia de investigación". Centro de Estudios de Estado y Sociedad (CEDES), Documento G.E. CLACSO.Vol. 4. En Lecturas sobre el Estado y las políticas públicas: Retomando el debate de ayer para fortalecer el actual. Buenos Aires. JGM. Presidencia de la Nación. Segunda Edición. 2008: 555-584.

Oszlak, Oscar (1977): "Notas criticas para una teoría de la burocracia estatal". Documento CEDES/G.E CLACSO.Vol. 8. Julio. Buenos Aires.

Oszlak, Oscar (1978): "Formación histórica del Estado en América Latina: elementos teórico-metodológicos para su estudio”. Estudios CEDES,Vol. 1, N 3. En Lecturas sobre el Estado y las políticas públicas: Retomando el debate de ayer para fortalecer el actual. Buenos Aires. JGM. Presidencia de la Nación. Segunda Edición. 2008: 115-142.

Oszlak, Oscar (1980): “Políticas públicas y regímenes políticos: reflexiones a partir de algunas experiencias latinoamericanas”, Estudios CEDES, Vol. 3, №2.

Oszlak, Oscar (2003): "El mito del estado mínimo: una década de reforma estatal en Argentina”. Desarrollo Económico, Buenos Aires, Argentina, vol. 42, 168: 33-61.

Oszlak, Oscar (2006): "Burocracia estatal: política y políticas públicas." POSTData Revista de Reflexión y Análisis Político. Buenos Aires, Argentina. 11:11-56

Quiroga, Hugo (2005): La Argentina en emergencia permanente. Buenos Aires: Edhasa.

Quiroga, Hugo (2010): La república desolada; los cambios políticos de la Argentina (20012009). Buenos Aires: Edhasa.

Sampay, Arturo Enrique (1951): "La Formación Política que la Constitución Argentina encarga a las Universidades". Boletín de Estudios Políticos. Instituto de Estudios Políticos. Universidad Nacional de Cuyo. Mendoza. Argentina. 1952 (2): 9-54. 
Sarlo, Beatriz (2007): La batalla de las ideas (1943-1973). Biblioteca del Pensamiento Argentino VII. Buenos Aires: Emecé.

Subirats, Joan et al. (2008): Análisis y gestión de políticas públicas. Barcelona: Ariel.

Thwaites Rey, Mabel (2001). “Tecnócratas vs punteros. Nueva falacia de una vieja dicotomía: política vs administración”. Encrucijadas, UBA, Buenos Aires, 6: 12-25.

Thwaites Rey, Mabel y López, Andrea (editoras) (2005). Entre tecnócratas globalizados y políticos clientelistas. Derrotero del ajuste neoliberal en el estado argentino. Buenos Aires: Prometeo.

\section{Documentos citados}

Alfonsín, Raúl (1985): “Discurso Parque Norte”. En Aznar, Luis y otros (1986): Discursos sobre el discurso. Buenos Aires. Eudeba-FUCADE. 11-41.

Diálogo Argentino. Boletín informativo. No 1.30 de enero de 2002.

http://www.presidenciaduhalde.com.ar/system/contenido.php?id_cat=37 (Recuperado 14 de octubre 2012).

Diario de Sesiones de la Convención Nacional Constituyente de 1949. Tomo I. Debates y Sanciones. 24 de enero - 16 de marzo. Buenos Aires. Imprenta del Congreso de la Nación.

Diario de Sesiones. Convención Nacional Constituyente. $15^{\text {a }}$ Reunión. $3^{\text {a }}$ Sesión Ordinaria. 22 de Julio de 1994

Diario de Sesiones. Convención Nacional Constituyente. $16^{\text {a }}$ Reunión. $3^{\text {a }}$ Sesión Ordinaria. 25 de Julio de 1994.

Escuela Nacional de Gobierno. Documento Inauguración Ciclo Lectivo 1998. Buenos Aires. Argentina.

Escuela Nacional de Gobierno. Informe anual de actividades 2002. Material aportado por el AG Enrique Bellagio. Febrero, 2012.

Instituto Nacional de Capacitación Política (2011): Informe de Gestión 2008-2011. Ministerio del Interior. Secretaría de Asuntos Políticos y Electorales. 
Jefatura de Gabinete de Ministros. Memoria Detallada del estado de la Nación Año 2002. Marzo 2003. http://www.jgm.gov.ar (Recuperado 20 de noviembre, 2011)

Jefatura de Gabinete de Ministros. Memoria Detallada del estado de la Nación Año 2003. Marzo 2004. http://www.jgm.gov.ar (Recuperado 23 de noviembre, 2011)

Jefatura de Gabinete de Ministros. Memoria Detallada del estado de la Nación Año 2011. Marzo 2012. http://www.jgm.gov.ar (Recuperado 23 de noviembre, 2011) 